\title{
SPECTRAL ESTIMATION BASED ON STRUCTURED LOW RANK MATRIX PENCIL
}

\author{
Javad Razavilar, Ye Li and K. J. Ray Liu \\ Electrical Engineering Department and Institute for Systems Research \\ University of Maryland, College Park, MD 20742 \\ (javad, liye, kjrliu@isr.umd.edu)
}

\begin{abstract}
This paper proposes a new parameter estimation algorithm for damped sinusoidal signals. Parameter estimation for damped sinusoidal signals with additive white noise is a problem of significant interest in many signal processing applications, like analysis of NMR data and system identification. The new algorithm estimates the signal parameters using a matrix pencil constructed from the measured data. To reduce the noise effect, rank deficient Hankel approximation of prediction matrix is used. The performance of the new algorithm is significantly improved by structured low rank approximation of prediction matrix. Computer simulations show that the noise threshold of the new algorithm is significantly better than the existing algorithms.
\end{abstract}

\section{INTRODUCTION}

High resolution parameter estimation for damped sinusoidal signals in the presence of additive white noise is a problem of significant interest in many signal processing applications, like spectral analysis, analysis of NMR data and system identification. Many approaches to high resolution parameter estimation have been proposed for pure sinusoidal signals, including linear prediction (LP) techniques [1], signal subspace methods introduced by Pisarenko, and generalized by Schmidt [2] in his MUSIC (multiple signal classification) algorithm and ESPRIT [3] which provides high resolution parameter estimation by means of subspace rotational invariance techniques with com-

This work was supported in part by the NIH grant 1R01GM49707 and the NSF grants MIP9309506 and MIP9457397. plexity much less than MUSIC algorithm. The difficulty of parameter estimation for damped sinusoidal signals stems from the nonstationarity of this kind of signals. Kumaresan-Tufts (KT) algorithm [4] and Hua-Sarkar's matrix pencil method [7] are well known to provide better estimations for parameters of damped sinusoidal signals. These two methods can attain CramerRao bound for a certain noise threshold if the damping factors are small.

A new algorithm is proposed in this paper which estimates the signal parameters by using a matrix pencil constructed from noise corrupted data. To reduce the noise effect, the low rank Hankel approximation of the prediction matrix is used $[5,6]$ . It can be shown that the performance of parameter estimation algorithm can be improved if the structure of the prediction matrix is preserved after low rank approximation. This structured low rank approximation of prediction matrix $[5,6]$ has a great effect on the performance of the new algorithm. The performance of the new algorithm is compared with $K T$ algorithm [4], modified $K T$ algorithm $(M K T)[6]$ and Hua-Sarkar's matrix pencil method [7] through the computer simulations. Computer simulation results show that, this new algorithm has lower noise threshold than $K T$ algorithm [4], MKT [6] and Hua-Sarkar's matrix pencil algorithms [7].

\section{ALGORITHM DEVELOPMENT}

Consider a sequence $y(n)$ consisted of $K$ damped sinusoidal signals and white noise $w(n)$ as

$$
y(n)=\sum_{k=1}^{K} c_{k} e^{s_{k} n}+w(n), \quad n=0, \cdots, N-1 .
$$


where $K$ is the number of damped sinusoids, $N \geq$ $2 K, s_{k}=-\alpha_{k}+\jmath \omega_{k}, \omega_{k} \in[-\pi, \pi], \alpha_{k} \geq 0$, which is called the damping factor and $w(n)$ represents the measurement noise. We first form $K \times K$ matrices as

$$
\mathbf{A}_{n}=\left(\begin{array}{lll}
y(n) & \cdots & y(n+K-1) \\
y(n+1) & \cdots & y(n+K) \\
\vdots & \vdots & \vdots \\
y(n+K-1) & \cdots & y(n+2 K-2)
\end{array}\right)
$$

for $n=0,1, \cdots, N-2 K+1$. From (1) it can be shown that

$$
\mathbf{A}_{n}=\mathbf{S}^{T} \mathbf{C} \boldsymbol{\Phi}^{n} \mathbf{S}+\mathbf{W}_{n},
$$

where in the above expression $\mathbf{W}_{n}$ 's are noise matrices which have the same structure as data matrices $\mathbf{A}_{n}, \mathbf{\Phi}$ and $\mathbf{C}$ are diagonal matrices defined as $\boldsymbol{\Phi}=\operatorname{diag}\left(e^{s_{1}}, e^{s_{2}}, \cdots, e^{s_{K}}\right), \mathbf{C}=$ $\operatorname{diag}\left(c_{1}, c_{2}, \cdots, c_{K}\right), \mathbf{S}$ is the signal matrix defined as $\mathbf{S}=\left[\mathbf{r}\left(s_{1}\right), \mathbf{r}\left(s_{2}\right), \cdots, \mathbf{r}\left(s_{K}\right)\right]^{T}$, where $\mathbf{r}\left(s_{k}\right)=\left[1, e^{s_{k}}, \cdots, e^{(K-1) s_{k}}\right]^{T}$.

If there is no measurement noise:

$$
\mathbf{A}_{n}^{-1} \mathbf{A}_{n+1}=\mathbf{S}^{-1} \mathbf{\Phi} \mathbf{S} \text {. }
$$

Therefore the eigenvalues of $\mathbf{A}_{n}^{-1} \mathbf{A}_{n+1}$ are $e^{s_{1}}, e^{s_{2}}, \cdots, e^{s_{K}}$, so signal parameters can be estimated from eigenvalues of $\mathbf{A}_{n}^{-1} \mathbf{A}_{n+1}$. Due to the existence of noise, we can only estimate $\mathbf{P}=\mathbf{S}^{-1} \mathbf{\Phi S}$. To obtain an accurate estimation of $\mathbf{P}$, we construct the following statistics

$$
\widehat{\mathbf{P}}=\sum_{n=0}^{N-2 K} a_{n} \mathbf{A}_{n}^{-1} \mathbf{A}_{n+1},
$$

where $a_{n}$ 's are the weighting factors. To make the estimation unbiased we must ensure $\sum_{n=0}^{N-2 K} a_{n}=$ 1. By selecting $a_{n}$ properly a good estimation of $\widehat{\mathbf{P}}$ can be obtained, therefore the performance can be enhanced under the influence of noise.

\section{HANKEL APPROXIMATION}

In the presence of measurement noise, we have to reduce the noise effect before we can apply the above algorithm. In other words, we have to first approximate noisy sequence $y(n)$ with sequence $\widehat{y}(n)$ which is less noisy, and then apply the new matrix pencil algorithm to the $\widehat{y}(n)$. For this purpose we first construct a prediction matrix from the measured data as

$$
\mathbf{A}_{y}=\left(\begin{array}{llll}
y(0) & y(1) & \ldots & y(L-1) \\
y(1) & y(2) & \ldots & y(L) \\
\vdots & \vdots & \vdots & \vdots \\
y(L-1) & y(L) & \ldots & y(2 L-2)
\end{array}\right)
$$

where $L=\lceil N / 2\rceil$ in order to have the best performance. Rank deficient Hankel approximation of the $\mathbf{A}_{y}$ given in $[5,6]$ is used to find the sequence $\widehat{y}(n)$ as follows:

Initialization: $\widehat{\mathbf{A}}_{y}^{[0]}=\mathbf{A}_{y}$ and $r=0$ ( $\mathrm{r}$ is the iteration index)

1) Compute $\quad S V D\left(\hat{\mathbf{A}}_{y}^{[r]}\right)=\mathbf{U D V}^{H}$

2) Obtain $\overline{\mathbf{A}}_{y}=\left[\bar{y}_{i, j}\right]_{i, j=0}^{L-1}=\sum_{k=1}^{K} \sigma_{k} \mathbf{u}_{k} \mathbf{v}_{k}^{H}$.

3) Find a Hankel matrix $\widehat{\mathbf{A}}_{y}^{[r]}$ to minimize $\|$ $\widehat{\mathbf{A}}_{y}^{[r]}-\overline{\mathbf{A}}_{y} \|_{F}$, (the best least square fit to $\overline{\mathbf{A}}_{y}$ )

$$
\widehat{\mathbf{A}}_{y}^{[r]}=\left[\widehat{y}^{[r]}(i+j)\right]_{i, j=0}^{L-1},
$$

where

$$
\widehat{y}_{i+j}^{[r]}=\frac{1}{\Gamma} \sum_{n+m=i+j, 0 \leq n, m \leq L-1} \bar{y}_{n, m} .
$$

in which $\Gamma$ is the number of the elements in matrix $\overline{\mathbf{A}}_{y}$ satisfying $n+m=i+j$ in (8).

4) Repeat steps 1, 2 and 3 till the rank of $\widehat{\mathbf{A}}_{y}^{[r]}=K$ (where $\mathrm{K}$ is the number of signals).

In [5] it is formally proved that this process converges to a rank deficient Hankel matrix. The sequence $\widehat{y}(n)$ resulted from the above rank deficient Hankel approximation algorithm is used in the new algorithm instead of $y(n)$ to estimate the signal parameters effectively.

Using the above Hankel approximation method for noise reduction in our new matrix pencil algorithm we have shown that the best weighting factors $a_{n}$ in (5) are as follows

$$
a_{n}=\frac{\left|\operatorname{det}\left(\mathbf{A}_{n}\right)\right|^{2 / K}(L+1-|L-n|)}{\sum_{k}\left|\operatorname{det}\left(\mathbf{A}_{k}\right)\right|^{2 / K}(L+1-|L-k|)} .
$$




\section{SIMULATION EXAMPLES}

In this section, we will demonstrate the performance of the new matrix pencil algorithm by two examples drawn from [4].

\section{Example 1:}

The simulated data are generated by

$y(n)=e^{s_{1} n}+e^{s_{2} n}+w(n), \quad$ for $n=0,1, \cdots, 24$.

where $s_{1}=-0.1+\jmath 2 \pi(0.52), s_{2}=-0.2+$ $\jmath 2 \pi(0.42), w(n)$ is complex white Gaussian noise with zero-mean and variance $\sigma^{2}$, related to $S N R$ by $\mathrm{SNR}=10 \log \left(\frac{1}{2 \sigma^{2}}\right)$. The MSE's of $\omega_{1}, \alpha_{1}, \omega_{2}$ and $\alpha_{2}$ for KT [4] algorithm, MKT [6], HuaSarkar's matrix pencil method [7] and the new algorithm, using the average of 500 trails, are shown in Figure 1. From Figure $1(a)$, the noise threshold for estimated frequency associated with smaller damping factor has lower noise threshold than the estimated frequency associated with larger damping factor, Figure $1(c)$. For $\omega_{1}$ with damping factor $\alpha_{1}=0.1$, from Figure $1(a)$, the noise threshold of the new matrix pencil algorithm is about $12 d B$ lower than $\mathrm{KT}$ algorithm and about $8 d B$ is lower than MKT and HuaSarkar's matrix pencil algorithms. For $\omega_{2}$ with damping factor $\alpha_{1}=0.2$, from Figure $1(c)$, the noise threshold of the new matrix pencil algorithm is about $10 \mathrm{~dB}$ lower than $\mathrm{KT}$ algorithm and about $5 d B$ is lower than MKT and HuaSarkar's matrix pencil algorithms.

Example 2:

We are going to estimate the poles and zeros of a linear system from its noise corrupted samples of the impulse response. The transfer function of the linear system is

$$
H(z)=\frac{B(z)}{A(z)}=\frac{1+\sum_{k=1}^{2} b_{k} z^{-k}}{1+\sum_{k=1}^{10} a_{k} z^{-k}}
$$

where poles of the transfer function are shown in Table 1. The magnitude of $H\left(e^{j \omega}\right)$ is shown in Figure 2 (a), which has two nulls at $\omega= \pm \pi / 4$ respectively.

The first forty real valued samples of noise corrupted impulse response are observed. The noise is real white Gaussian with zero-mean and variance $\sigma^{2}$ determined by SNR, defined as

$$
S N R=10 \log \left(\frac{\sum_{n=0}^{39}|h(n)|^{2}}{N \sigma^{2}}\right),
$$

KT algorithm, MKT algorithm and the new algorithm are applied to estimate the poles of the system, consequently, the denominator of $\widehat{A}(z)$. Once $\widehat{A}(z)$ is obtained, $B(z)$ can be estimated using Shanks' method [8], which first generates a sequence $f_{n}$ by

$$
f(n)=\mathcal{Z}^{-1}\left\{\frac{1}{\widehat{A}(z)}\right\},
$$

and then estimate $b_{k}$ for $k=0,1,2$ by minimizing the error

$$
E=\sum_{n=0}^{39}\left|h(n)-\sum_{k=0}^{2} \widehat{b}_{k} f(n-k)\right|^{2} .
$$

Figure 2 (b), (c) and (d) show 10 trails of magnitudes of estimated transfer function using $\mathrm{KT}$, $\mathrm{MKT}$ and the new matrix pencil algorithms respectively. Also Table 1 illustrates the mean and variance of the estimated poles of transfer function using $\mathrm{KT}, \mathrm{MKT}$ and the new matrix pencil algorithms. From Figure 2 and Table 1, it is clear that the new matrix pencil algorithm gives more accurate estimate of transfer function $H(z)$ than $\mathrm{KT}$ and MKT algorithms.

\section{CONCLUSION}

In this paper a novel parameter estimation algorithm for exponentially damped sinusoidal signals is proposed. Signal parameters are estimated by a matrix pencil constructed from the given data. The noise effect is reduced significantly by using low rank Hankel approximation of prediction matrix. Computer simulations demonstrate that the new algorithm can estimate the parameters of exponentially damped sinusoids effectively.

\section{REFERENCES}

[1] S. Haykin, Adaptive Filter Theory, Prentice Hall, 1991.

[2] R. O. Schmidt, "Multiple Emitter Location and Signal Parameter Estimation" IEEE Trans. on Ant. and Prop., March 1986. 
[3] R. Roy, T. Kailath, "ESPRIT- Estimation of Signal Parameters Via Rotational Invariance Techniques," IEEE Trans. on ASSP, Jul. 1989.

[4] R. Kumaresan and R. W.Tufts, "Estimation the parameters of exponentially damped sinusoids and pole-zero modeling in noise," IEEE Trans. on ASSP, Dec. 1982.

[5] J. Cadzow, "Signal Enhancement: A Composite property Mapping Algorithm," IEEE Trans. on Acoust., Speech, Signal Processing, Oct. 1988.

[6] Y. Li, K. J. R. Liu, J. Razavilar, "Improved parameter estimation schemes for damped sinusoidal signals," Conference on Information Sciences and Systems (CISS), John Hopkins University, March 1995.

[7] Y. Hua, T. K. Sarkar, "Matrix Pencil Method for Estimating Parameters of Exponentially Damped/Undamped Sinusoids in Noise", IEEE Trans. on ASSP, pp 814-824, May 1990

[8] J. L. Shanks, "Recursion filters for digital processing," Geophysics, vol.32, pp.33-51, 1967.
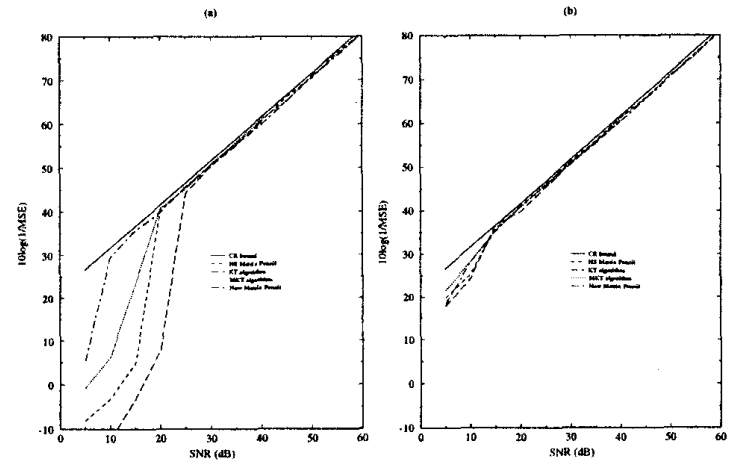

(c)
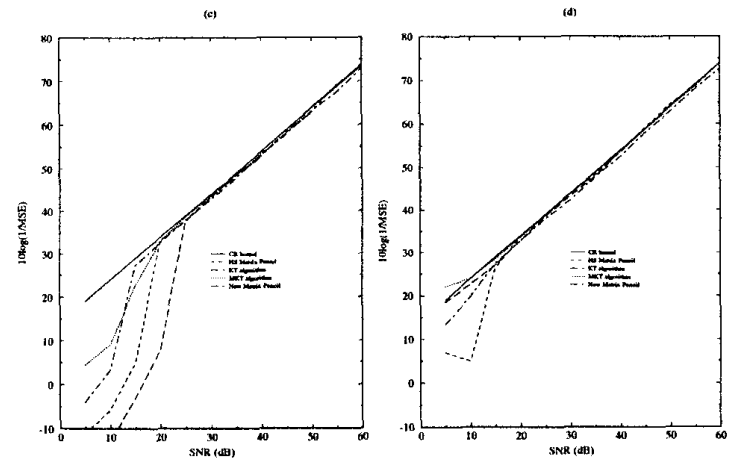

Figure 1. The MSE of (a) $\omega_{1}$, (b) $\alpha_{1}$, (c) $\omega_{2}$ (d) $\alpha_{2}$ for $K T$, MKT, Hua-Sarkar's matrix pencil and the new matrix pencil algorithms when $s_{1}=-0.1+\jmath 2 \pi 0.52, s_{2}=-0.2+\jmath 2 \pi 0.42$ and $N=25$.
Table 1. The true and estimated poles of the transfer function $H(z)$

\begin{tabular}{|c|c|c|c|c|}
\hline \multirow[t]{2}{*}{ True Poles } & \multirow{2}{*}{\multicolumn{2}{|c|}{ Method }} & \multicolumn{2}{|c|}{ Estimated Poles } \\
\hline & & & $S N R=30 d B$ & $S N R=20 d B$ \\
\hline \multirow{6}{*}{$\begin{array}{r}-0.2913 \\
\pm ر 0.8968\end{array}$} & KT & Mean & $-0.2880 \pm, 0.9080$ & $-0.3448 \pm, 0.8720$ \\
\hline & alg. & Std. & $1.498 \times 10^{-4}$ & $8.583 \times 10^{-2}$ \\
\hline & MKT & Mean & $-0.2915 \pm 0.8970$ & $-0.2917 \pm, 0.8976$ \\
\hline & alg. & Std. & $3.762 \times 10^{-5}$ & $3.376 \times 10^{-}$ \\
\hline & New & Mean & $-0.2914 \pm 0.8964$ & $-0.2914 \pm, 0.8959$ \\
\hline & alg. & Std. & $1.048 \times 10^{-5}$ & $1.088 \times 10^{-4}$ \\
\hline \multirow{6}{*}{$\begin{array}{c}0.1014 \\
\pm ر 0.9579\end{array}$} & KT & Mean & $0.0987 \pm 00.9510$ & $0.0657 \pm, 0.9392$ \\
\hline & aig. & Std. & $1.713 \times 10^{-5}$ & $1.406 \times 10^{-2}$ \\
\hline & MKT & Mean & $0.1015 \pm 30.9577$ & $0.1015 \pm 20.9571$ \\
\hline & alg. & Std. & $4.311 \times 10^{-6}$ & $4.556 \times 10^{-5}$ \\
\hline & New & Mean & $0.1014 \pm 0.9580$ & $0.1014 \pm, 0.9578$ \\
\hline & alg. & Std. & $5.800 \times 10^{-7}$ & $6.292 \times 10^{-6}$ \\
\hline \multirow{6}{*}{$\begin{array}{c}0.2959 \\
\pm ر 0.9292\end{array}$} & $\overline{K T}$ & Mean & $0.2979 \pm 0.0232$ & $0.2845 \pm 20.9183$ \\
\hline & alg. & Std. & $2.784 \times 10^{-5}$ & $3.858 \times 10^{-3}$ \\
\hline & MKT & Mean & $0.2960 \pm j 0.9291$ & $0.2959 \pm ر 0.9287$ \\
\hline & aig. & Std. & $1.992 \times 10^{-6}$ & $1.607 \times 10^{-5}$ \\
\hline & New & Mean & $0.2959 \pm, 0.9292$ & $0.2960 \pm, 0.8292$ \\
\hline & alg. & Std. & $2.295 \times 10^{-7}$ & $2.211 \times 10^{-6}$ \\
\hline \multirow{6}{*}{$\begin{array}{c}0.5630 \\
\pm ر 0.8019\end{array}$} & $\mathrm{KT}$ & Mean & $0.5620 \pm, 0.8175$ & $0.5339 \pm, 0.8442$ \\
\hline & alg. & Std. & $1.827 \times 10^{-4}$ & $8.359 \times 10^{-3}$ \\
\hline & MKT & Mean & $0.5629 \pm j 0.8023$ & $0.5626 \pm, 0.8045$ \\
\hline & alg. & Std. & $1.059 \times 10^{-5}$ & $9.462 \times 10^{-5}$ \\
\hline & New & Mean & $0.5629 \pm, 0.8018$ & $0.5629 \pm 0.8020$ \\
\hline & alg. & Std. & $2.224 \times 10^{-6}$ & $2.233 \times 10^{-5}$ \\
\hline \multirow{6}{*}{$\begin{array}{c}0.9815 \\
\pm j 0.1117\end{array}$} & KT & Mean & $0.9853 \pm, 0.1089$ & $0.9798 \pm 0.1219$ \\
\hline & alg. & Std. & $2.120 \times 10^{-5}$ & $1.763 \times 10^{-2}$ \\
\hline & MKT & Mean & $0.9816 \pm 00.1117$ & $0.9821 \pm, 0.1114$ \\
\hline & alg. & Std. & $1.894 \times 10^{-}$ & $1.811 \times 10^{-5}$ \\
\hline & New & Mean & $0.9815 \pm, 0.1118$ & $0.9815 \pm 00.1117$ \\
\hline & alg. & Std. & $5.858 \times 10^{-}$ & $4.988 \times 10^{-6}$ \\
\hline
\end{tabular}
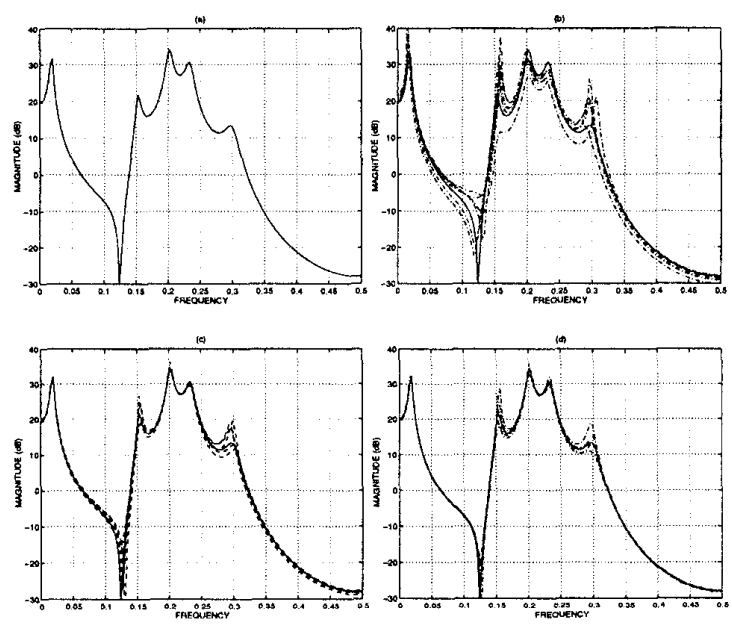

Figure 2. (a) Magnitude of $H(z)$, (b) estimated magnitude of $\widehat{H}(z)$ using KT algorithm, (c) estimated magnitude of $\widehat{H}(z)$ using MKT algorithm, (d) estimated magnitude of $\widehat{H}(z)$ using the new algorithm, $S N R=30 \mathrm{~dB}$. 